Supporting Information:

\title{
Selective Electrochemical Detection of Ciprofloxacin with a Porous Nafion/Multi-Walled Carbon Nanotube Composite Film Electrode
}

\author{
Pralay Gayen, ${ }^{\mathrm{a}}$ Brian P. Chaplin, ${ }^{\mathrm{a}, ~} *$ \\ ${ }^{a}$ Department of Chemical Engineering, University of Illinois at Chicago, Chicago, IL 60607, \\ USA
}

Corresponding author:

Phone No.: +13129960288, E-mail: chaplin@uic.edu (Brian P. Chaplin)

Pages: 9

Tables: 1

Figures: 12 

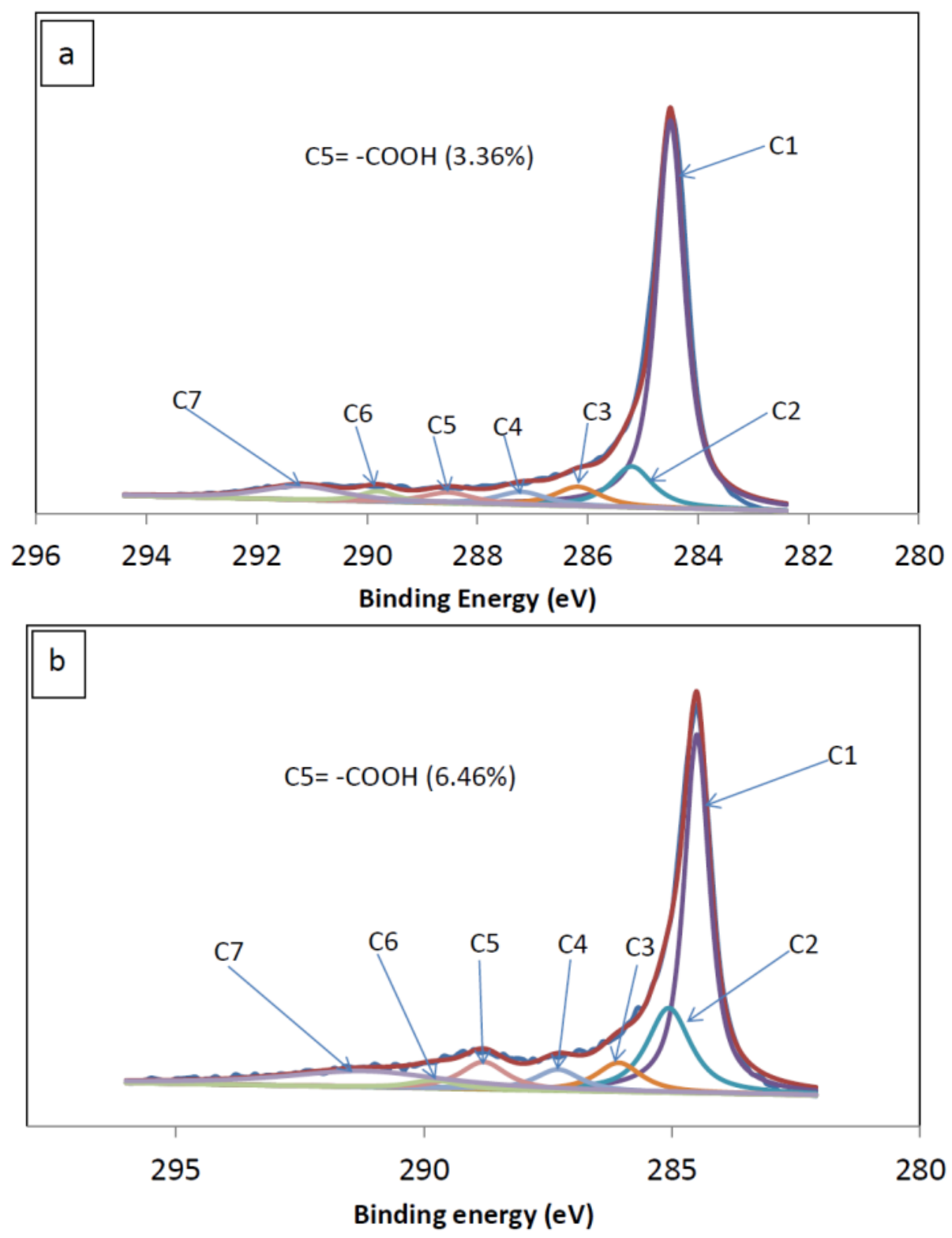

Figure S-1. XPS of (a) MWCNT and (b) MWCNT-O (oxidized sample). Legend: $\mathrm{C} 1$ : C=C; C2: C-C; C3: $-\mathrm{C}-\mathrm{OH}$; $\mathrm{C} 4:-\mathrm{C}=\mathrm{O}$; C5: $-\mathrm{COOH}$; $\mathrm{C} 6:-\mathrm{CO}_{3} ; \mathrm{C}$ : $\pi-\pi^{*}$ 

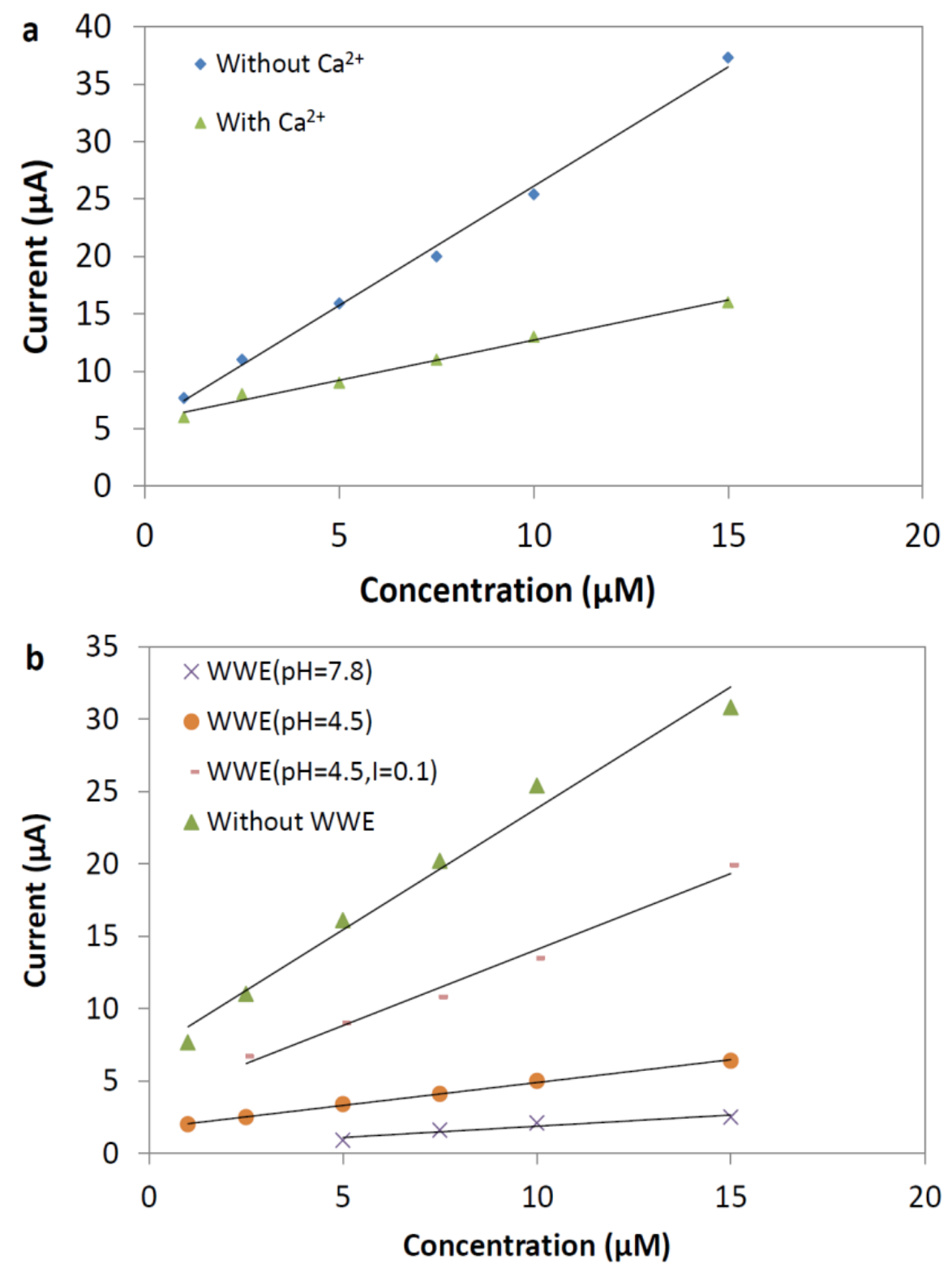

Figure S-2. (a) Calibration graphs at different concentration of CFX with (slope=

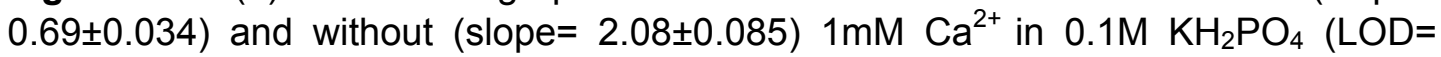
$0.005 \mu \mathrm{M}$, linear ranges $(\mu \mathrm{M})=0.05-1,1-15)$, (b) Calibration graphs at different concentration of CFX without $\left(y=1.6768 \pm 0.11 x+7.0689 \pm 0.911, R^{2}=0.9831\right)$, with $\left(\mathrm{y}=0.1543 \pm 0.03 \mathrm{x}+0.3286 \pm 0.341, \mathrm{R}^{2}=0.9119\right), \mathrm{pH}(4.5)$ adjusted $(\mathrm{y}=$ $0.3158 \pm 0.007 x+1.7424 \pm 0.059, R^{2}=0.998$ and both $\mathrm{pH}(4.5)$ and conductivity $(0.1$ $\mathrm{M}$ electrolyte) adjusted $\left(y=1.0492 \pm 0.07 x+3.5865 \pm 0.636, R^{2}=0.9868\right)$ wastewater effluent matrix. 


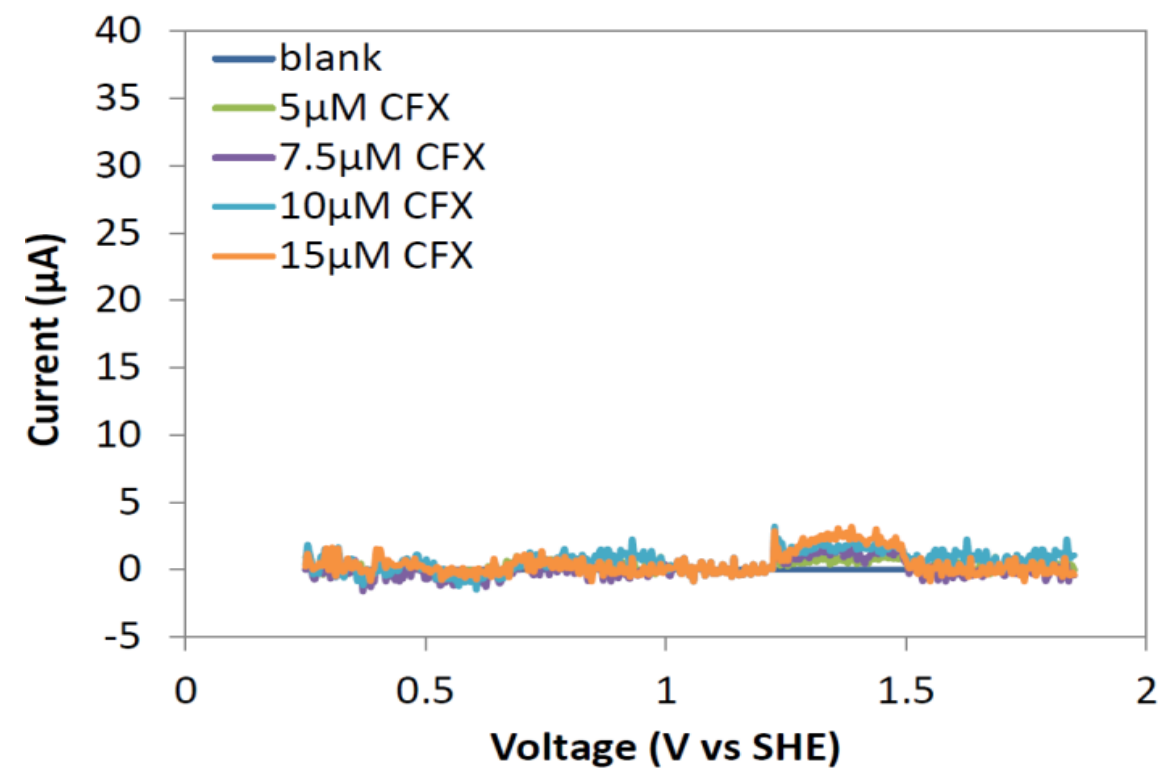

Figure S-3. DPV curves of porous-Nafion-MWCNT/BDD at different concentrations of CFX with WWE $(\mathrm{pH}=7.80)$.

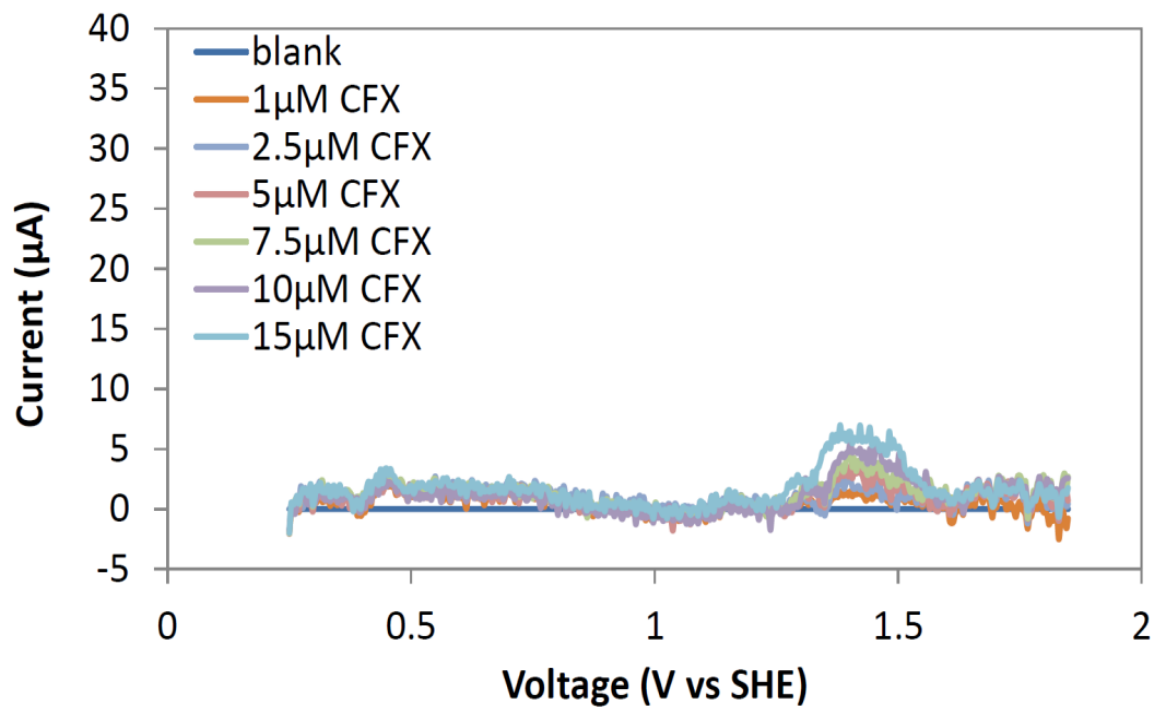

Figure S-4. DPV curves of porous-Nafion-MWCNT/BDD at different concentrations of CFX with WWE $(\mathrm{pH}=4.50)$. 


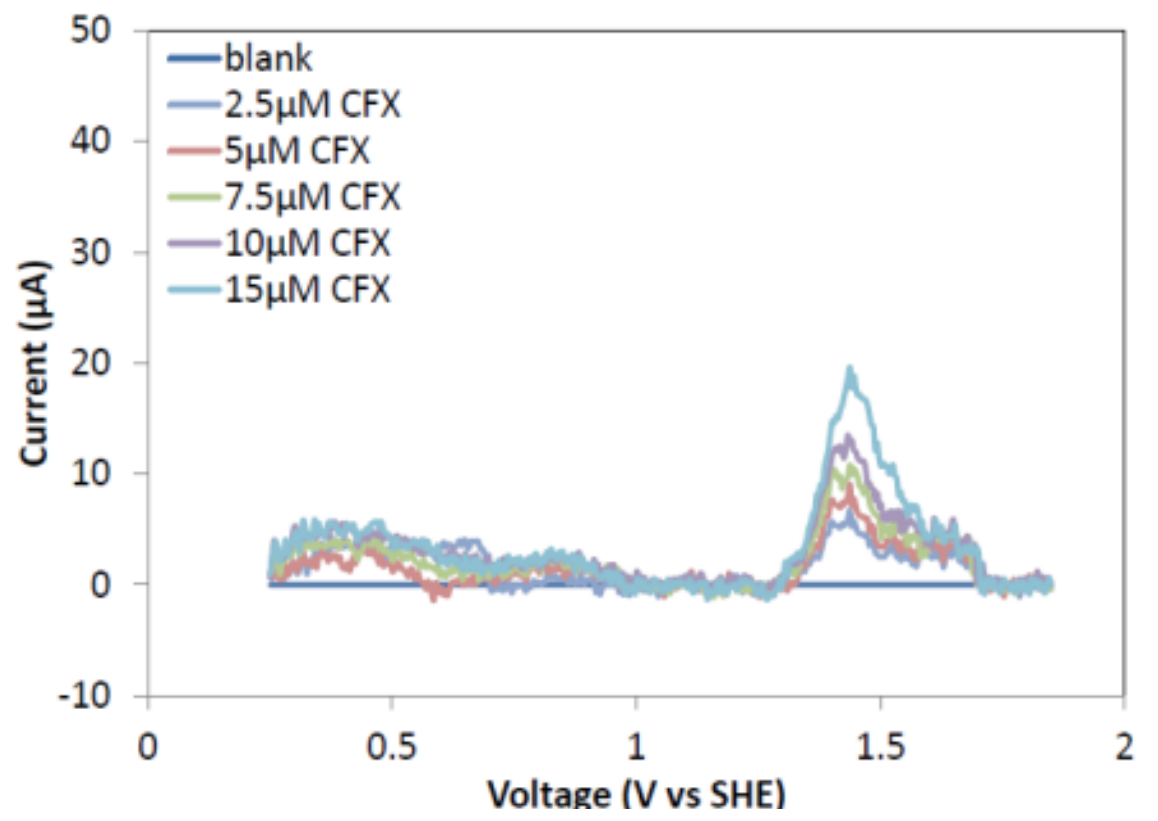

Figure S-5. DPV curves of porous-Nafion-MWCNT/BDD at different concentrations of CFX with WWE $\left(\mathrm{pH}=4.50 \& 0.1 \mathrm{M} \mathrm{KH}_{2} \mathrm{PO}_{4}\right)$.

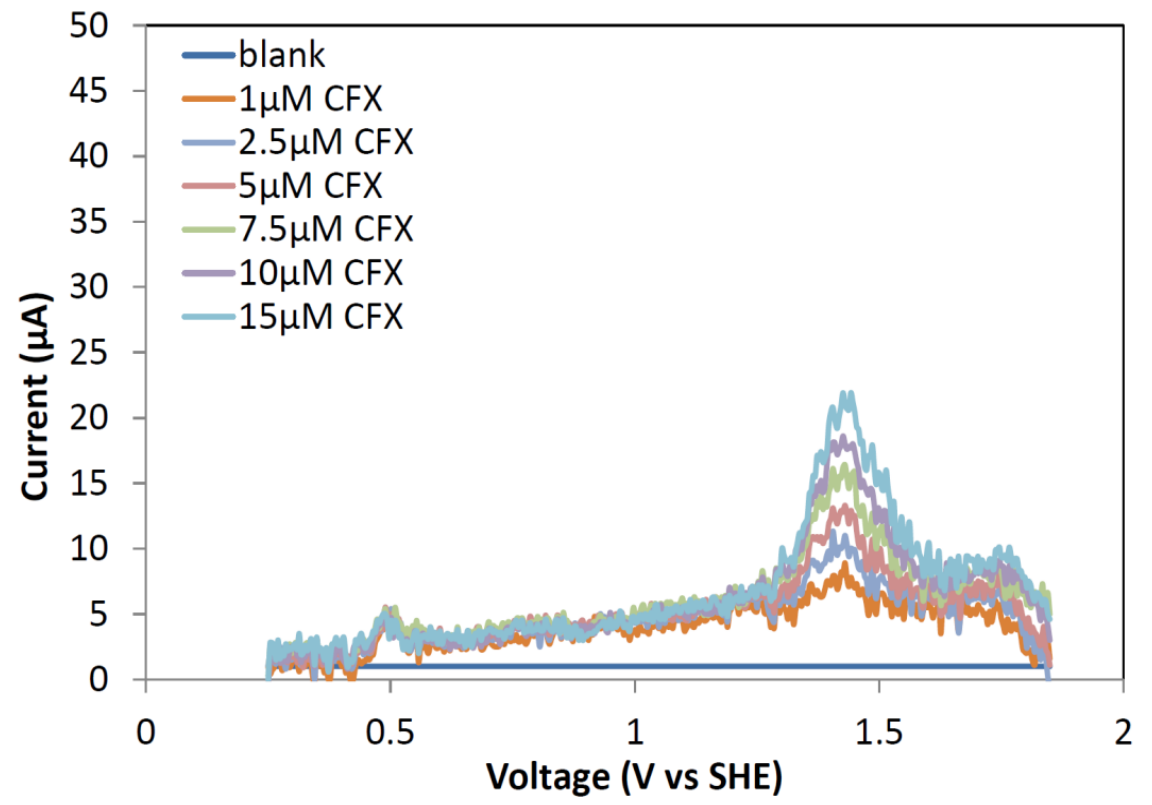

Figure S-6. DPV curves of porous-Nafion-MWCNT/BDD at different concentrations of CFX in $0.1 \mathrm{M} \mathrm{KH}_{2} \mathrm{PO}_{4}$ without organics. 


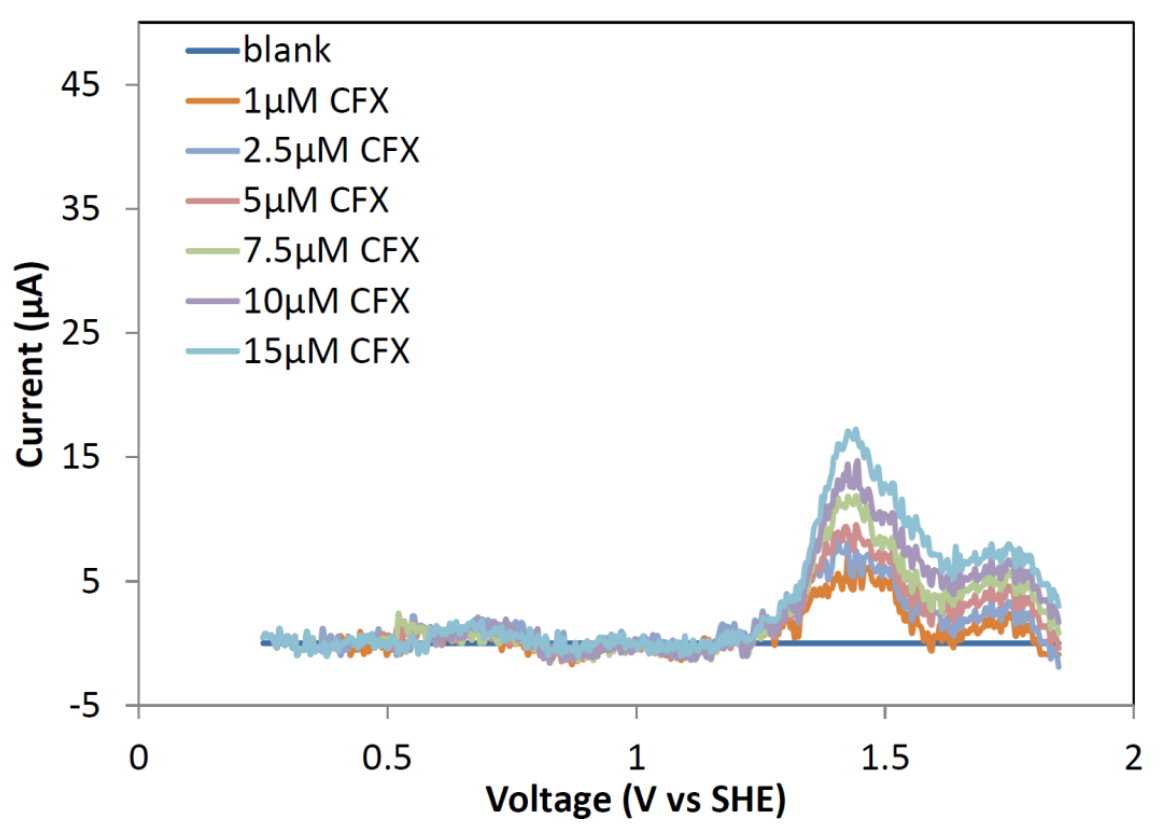

Figure S-7. DPV curves of porous-Nafion-MWCNT/BDD at different concentrations of CFX in $0.1 \mathrm{M} \mathrm{KH}_{2} \mathrm{PO}_{4}$ with 1:1 HBA.

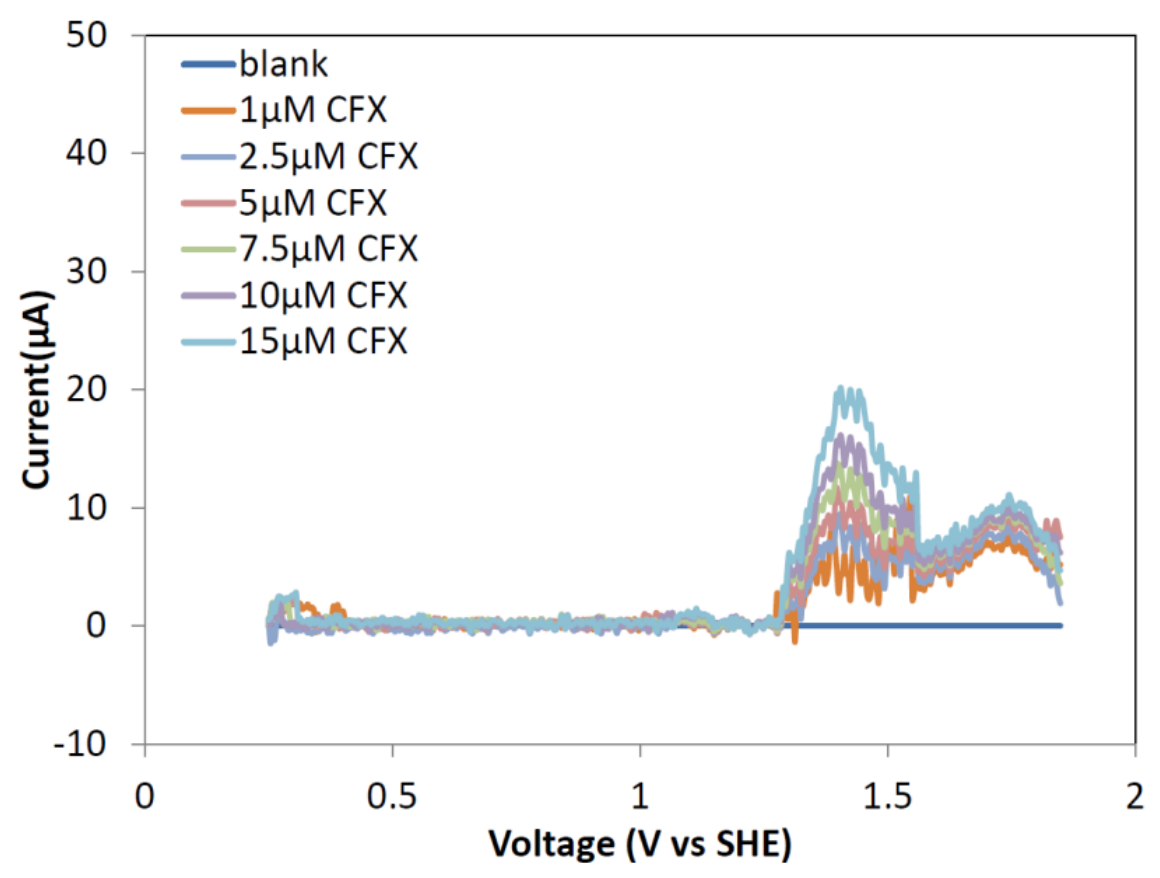

Figure S-8. DPV curves of porous-Nafion-MWCNT/BDD at different concentrations of CFX in $0.1 \mathrm{M} \mathrm{KH}_{2} \mathrm{PO}_{4}$ with 1:1 $\mathrm{ABA}$. 


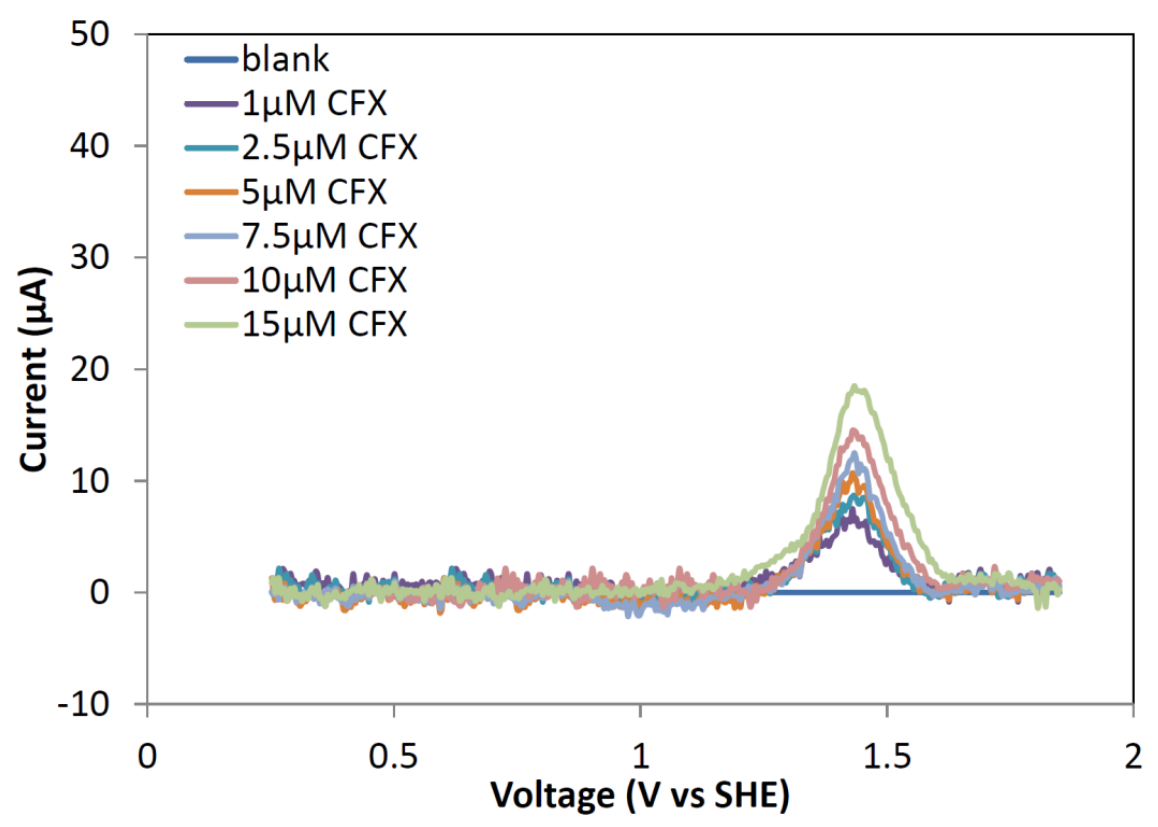

Figure S-9. DPV curves of porous-Nafion-MWCNT/BDD at different concentrations of CFX in $0.1 \mathrm{M} \mathrm{KH}_{2} \mathrm{PO}_{4}$ with 1:1 SA.

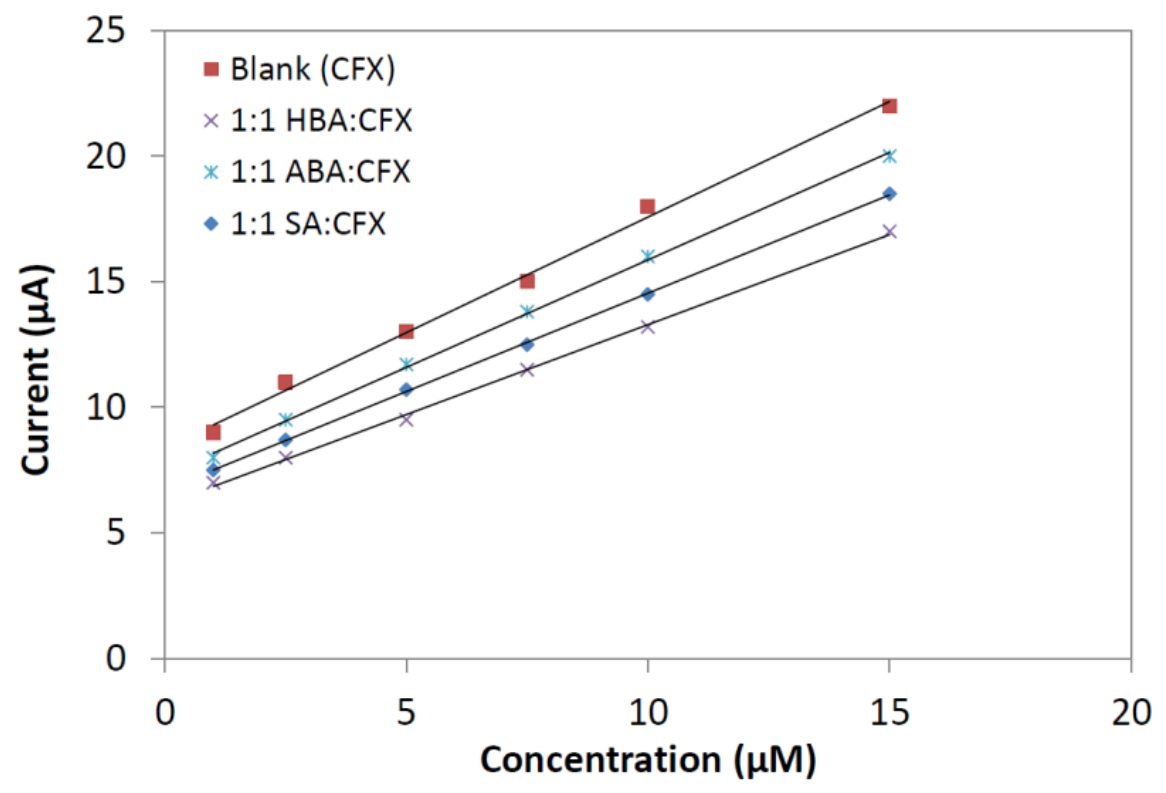

Figure S-10. Calibration graphs of CFX at different concentration without organics (blank/CFX only) $\left(y=0.920 \pm 0.03 x+8.38 \pm 0.249, R^{2}=0.996\right)$, with equimolar ABA ( $y$ $\left.=0.854 \pm 0.012 x+7.328 \pm 0.105, R^{2}=0.99\right)$, equimolar HBA $(y=0.72 \pm 0.052 x+$ $\left.6.144 \pm 0.112, R^{2}=0.999\right)$ and equimolar $S A\left(y=0.782 \pm 0.005 x+6.723 \pm 0.046, R^{2}=\right.$ 0.998). 


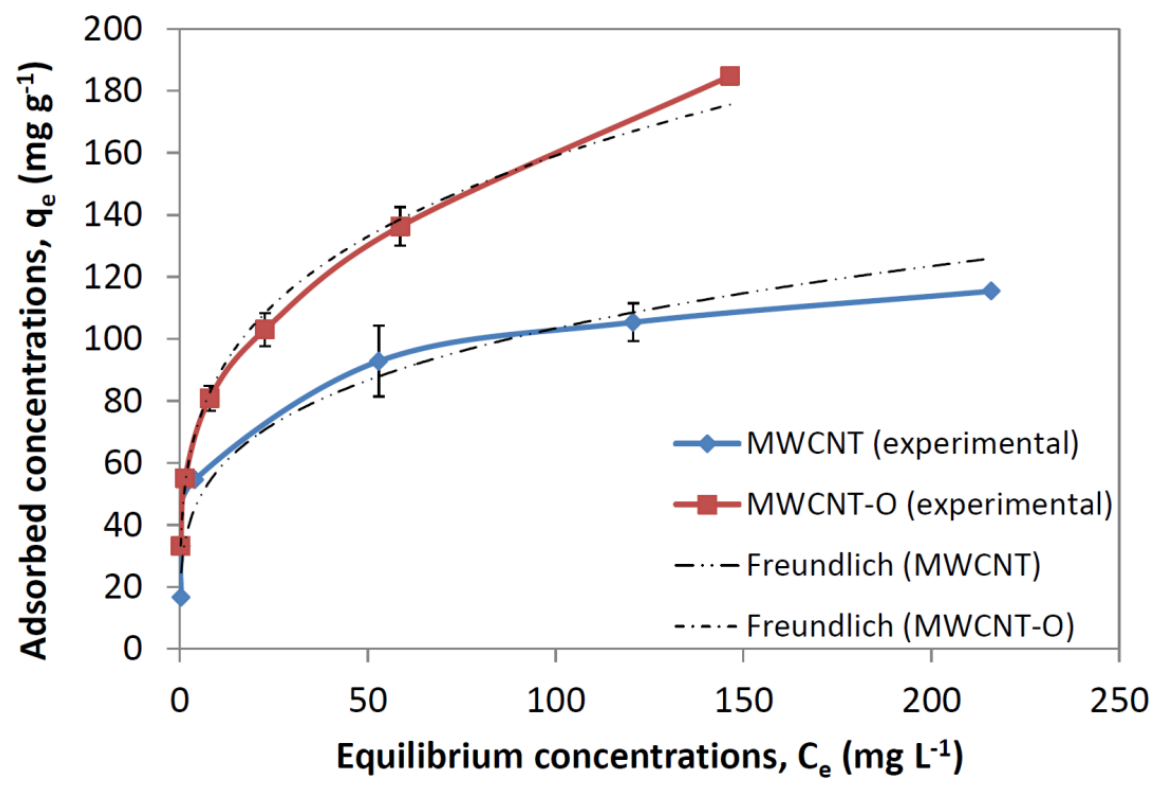

Figure S-11. Adsorption isotherms of ciprofloxacin on MWCNT and MWCNT-O at $20^{\circ} \mathrm{C}$ fitted with two models (Freundlich and Langmuir model).

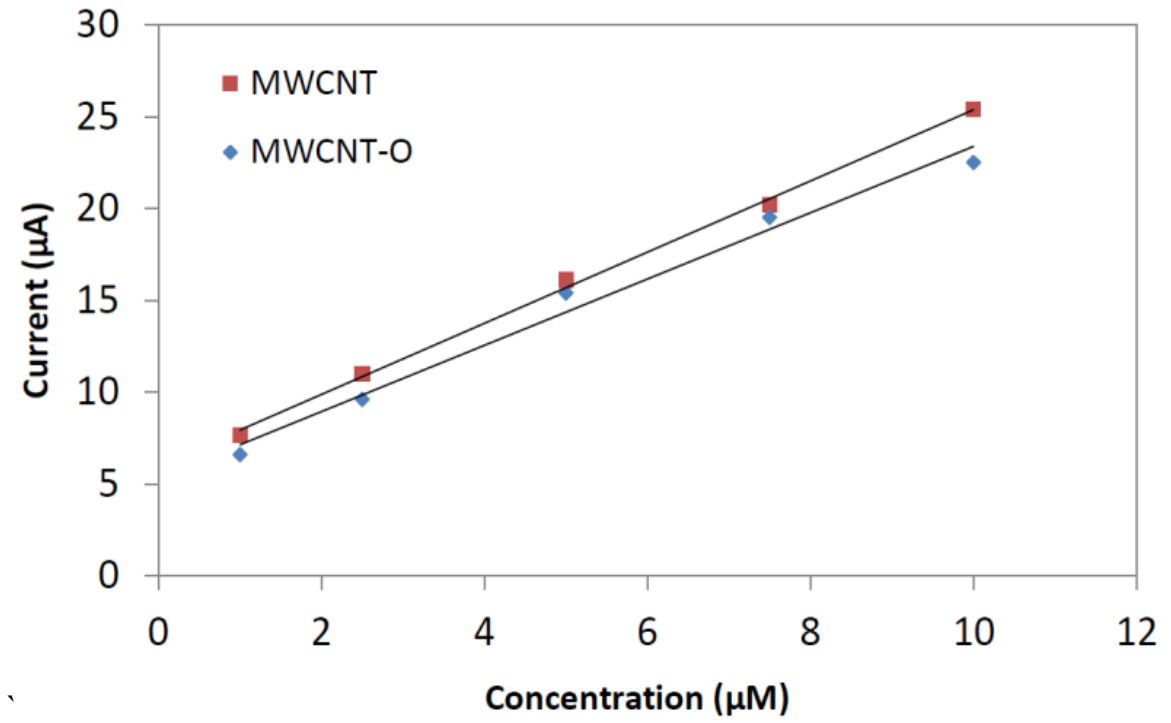

Figure S-12. Calibration graphs of CFX with porous-Nafion-MWCNT/BDD $(y=$ $1.9379 \pm 0.049 x+5.995 \pm 0.300, R^{2}=0.9981$, and porous-Nafion-MWCNT-O/BDD $\left(y=1.8036 \pm 0.13 x+5.341 \pm 0.784, R^{2}=0.9852\right)$. 
Table S-1 - Determination of CFX in the presence of different interferences (LDRs including LODs). Errors on estimates represent the standard errors.

\begin{tabular}{|c|c|c|c|}
\hline Interferences & $\begin{array}{l}\text { LOD } \\
(\mu \mathrm{M})\end{array}$ & $\begin{array}{c}\text { Linear range } \\
(\mu \mathrm{M})\end{array}$ & $\begin{array}{c}\text { Sensitivity } \\
\left(\text { Slope }\left(\mu A \mu M^{-1}\right), R^{2} \text {, Intercept) }\right.\end{array}$ \\
\hline No interference & 0.005 & $0.005-0.05$ & $\begin{array}{l}\text { Slope }=41 \pm 5.196, R^{2}=0.98 \\
\text { Intercept= } 2.055 \pm 0.153\end{array}$ \\
\hline $\mathrm{Ca}^{2+}$ & 0.005 & $0.005-1$ & $\begin{array}{l}\text { Slope }=4.15 \pm 0.849, R^{2}=0.89 \\
\text { Intercept }=1.9 \pm 0.425\end{array}$ \\
\hline Chloride (1mM) & 0.005 & $0.005-0.05$ & $\begin{array}{l}\text { Slope }=33.84 \pm 8.78, \mathrm{R}^{2}=0.94 \\
\text { Intercept= } 2.633 \pm 0.259\end{array}$ \\
\hline SDBS (1mM) & 0.005 & $0.005-0.05$ & $\begin{array}{l}\text { Slope }=23.25 \pm 7.83, R^{2}=0.907 \\
\text { Intercept }=0.565 \pm 0.232\end{array}$ \\
\hline Humic $\operatorname{acid}\left(11 \mathrm{mg} \mathrm{L}^{-1}\right)$ & 0.005 & $0.005-0.05$ & $\begin{array}{l}\text { Slope }=29.63 \pm 13.38, R^{2}=0.84 \\
\text { Intercept }=1.06 \pm 0.393\end{array}$ \\
\hline Amoxicillin (1mM) & 0.005 & $0.005-0.05$ & $\begin{array}{l}\text { Slope }=24.92 \pm 13.83, R^{2}=0.83 \\
\text { Intercept }=1.29 \pm 0.407\end{array}$ \\
\hline Without WWE & 0.005 & $0.005-0.05$ & $\begin{array}{l}\text { Slope }=38.61 \pm 10.53, R^{2}=0.93 \\
\text { Intercept= } 2.19 \pm 0.311\end{array}$ \\
\hline$W W E_{p H=4.5}$ & 0.005 & $0.005-0.05$ & $\begin{array}{l}\text { Slope }=11.09 \pm 4.98, R^{2}=0.84 \\
\text { Intercept }=0.459 \pm 0.147\end{array}$ \\
\hline$W W E_{p H=4.5, ~ l=0.1}$ & 0.005 & $0.005-0.05$ & $\begin{array}{l}\text { Slope }=13.42 \pm 4.74, R^{2}=0.901 \\
\text { Intercept }=0.442 \pm 0.14\end{array}$ \\
\hline WWE & 5 & $5-15$ & $\begin{array}{l}\text { Slope }=0.15 \pm 0.03, R^{2}=0.912 \\
\text { Intercept }=0.328 \pm 0.341\end{array}$ \\
\hline Without organics & 0.005 & $0.005-0.05$ & $\begin{array}{l}\text { Slope }=36.98 \pm 16.61, R^{2}=0.83 \\
\text { Intercept= } 2.198 \pm 0.491\end{array}$ \\
\hline 1:1 CFX:HBA & 0.005 & $0.005-0.05$ & $\begin{array}{l}\text { Slope }=36.43 \pm 14.73, R^{2}=0.85 \\
\text { Intercept= } 1.198 \pm 0.482\end{array}$ \\
\hline 1:10 CFX:HBA & 1 & $1-15$ & $\begin{array}{l}\text { Slope }=0.65 \pm 0.012, R^{2}=0.99 \\
\text { Intercept }=1.329 \pm 0.103\end{array}$ \\
\hline 1:1 CFX:ABA & 0.005 & $0.005-0.05$ & $\begin{array}{l}\text { Slope }=34.24 \pm 12.34, R^{2}=0.87 \\
\text { Intercept= 5.198 } \pm 0.861\end{array}$ \\
\hline 1:10 CFX:ABA & 0.05 & $0.05-1$ & $\begin{array}{l}\text { Slope }=2.10 \pm 0.064, R^{2}=0.999 \\
\text { Intercept }=3.832 \pm 0.845\end{array}$ \\
\hline
\end{tabular}

